\title{
Devulcanization of styrene butadiene rubber by microwave energy: Effect of the presence of ionic liquid
}

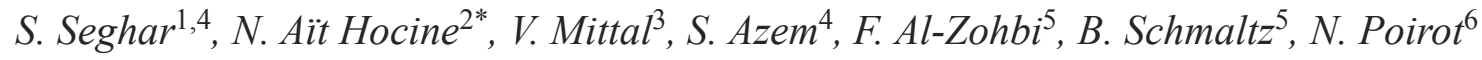 \\ ${ }^{1}$ LMR, CERMEL, Université François-Rabelais de Tours, 37300 Joué-Lès-Tours, France \\ ${ }^{2}$ LMR, INSA Centre Val de Loire, 3 rue de la Chocolaterie, BP 3410, 41034 Blois, France \\ ${ }^{3}$ Department of Chemical Engineering, The Petroleum Institute, Abu Dhabi, UAE \\ ${ }^{4}$ LEC2M, Université Mouloud Mammeri, BP 17, Tizi-Ouzou 15000, Algérie \\ ${ }^{5}$ PCM2E (E.A. 6299), Université François-Rabelais de Tours, Bâtiment J-Parc de Grandmont, 37200 Tours, France \\ ${ }^{6}$ GREMAN, IUT de Blois, 15 rue de la Chocolaterie, 41000 Blois, France
}

Received 25 April 2015; accepted in revised form 15 July 2015

\begin{abstract}
In this study, styrene butadiene rubber (SBR) was devulcanized using microwave irradiation. In particular, effect of ionic liquid (IL), pyrrolidinium hydrogen sulfate $[\mathrm{Pyrr}]\left[\mathrm{HSO}_{4}\right]$, on the devulcanization performance was studied. It was observed that the evolution of the temperature reached by rubber powder exposed to microwave irradiation for different energy values was favored by the presence of ionic liquid $[\mathrm{Pyrr}]\left[\mathrm{HSO}_{4}\right]$ significantly over the whole range of the microwave energy values. Beyond the threshold point of $220 \mathrm{Wh} / \mathrm{kg}$, the soluble fraction after devulcanization sharply increased with increasing devulcanization microwave energy. For the powder mixed with $[\mathrm{Pyrr}]\left[\mathrm{HSO}_{4}\right]$, the increase was more significant. Furthermore, the crosslink density was observed to decrease slowly with the microwave energy up to $220 \mathrm{Wh} / \mathrm{kg}$, beyond which the crosslink density decreased significantly for the rubber impregnated with IL. For the rubber with IL, significant and continuous increase in $T_{\mathrm{g}}$ with microwave energy values was observed in comparison with the SBR where no change in transition temperature was observed. Mechanical shearing of rubber gums in the two-roll mill favored the devulcanization process, which indicated that the combination of mechanical loading with microwave energy and IL is an efficient procedure allowing an optimal devulcanization of rubbers.
\end{abstract}

Keywords: rubber, recycling, devulcanization, microwaves, ionic liquid

\section{Introduction}

Increase in the demand of rubber in recent years, particularly in tire industry, has resulted in not only increased prices of raw materials, but also accumulation of harmful wastes. Garbage dumps of used tires represent flammable sites and generate proliferation of various insects and rodents carrying diseases. Moreover, slow degradation of rubber residues results in severe environmental pollution. Recycling such waste safely is therefore a big challenge for industrialists and academic researchers. Devulcanization of waste rubber products is one of such process which leads to recycling the waste tires as polymeric materials, thus, also decreasing the extent of environmental risks.

Passenger car tire material is a blend of different polymers with the main component being styrene-butadiene rubber (SBR). Each polymer contributing to the tire formulation has its own specific degradation and devulcanization characteristics. As a result, a number of physical and chemical methods have been developed to break the crosslink bonds or induce active groups on the surface of ground rubber. These methods are based on mechanical energy [1], ultra-

\footnotetext{
${ }^{*}$ Corresponding author, e-mail: nourredine.aithocine@insa-cvl.fr (C) BME-PT
} 
sound [2, 3], microwave irradiation [4, 5], chemical desulfurization reagents $[6,7]$ or biotechnological methods [8]. Depending on their efficacy, they lead to the devulcanization or the reclaiming process. Devulcanization targets the sulfuric crosslinks in the vulcanized rubber and thus carbon-sulfur $(\mathrm{C}-\mathrm{S})$ and sulfur-sulfur (S-S) bonds are selectively cleaved, while reclaiming is usually accompanied with considerable scission along the polymeric chains resulting in lower molecular mass fractions [9].

Microwave devulcanization process was proposed by Goodyear in 1978 and successfully implemented by Novotny et al. [4]. It consists of exposing the waste rubber, for a short time, to controlled microwave irradiation. However, only sulfur-vulcanized rubber containing polar groups or components can be heated and thus be devulcanized with this technique.

Microwave heating is based on the interaction of the oscillating electrical field of microwaves with the molecular dipoles and/or charged ions present in the sample. Microwave irradiation triggers heating by three main mechanisms: dipolar polarization, ionic conduction and interfacial polarization. While the dipolar polarization mechanism (dielectric heating) explains the heating phenomena of dipoles, the ionic conduction mechanism explains the heating in samples with free ions or ionic species. The interfacial polarization (also called the MaxwellWagner effect) mechanism describes the heating in non-homogeneous systems created by the suspension of conducting particles in a non-conducting medium. Fix [10] extensively studied the efficiency of the microwave devulcanization process and such operation was concluded to be feasible.

Other studies by Goodyear society on the devulcanization of EPDM rubber by microwaves showed that properties of the treated material were not considerably altered $[4,10]$. EPDM being a non-polar rubber, microwave energy was transferred in the whole volume of the material via carbon black filler initially introduced as reinforcing particles.

Bani et al. [11] also performed similar studies and observed that the microwave devulcanization process was effective only beyond the heating temperature of $300^{\circ} \mathrm{C}$.

Landini et al. [12] used a microwave technique to devulcanize bromobutyle (BIIR), and optimal power and time of treatment were identified as factors lead- ing to the highest devulcanization proportion. Similarly, Scagliusi et al. [13] devulcanized chloroprene rubber, followed by re-vulcanization. The authors observed that properties of the recycled material changed with microwave irradiation time, as compared to the original material. Hardness and fracture resistance were observed to decrease, whereas rupture strain increased. Vega et al. [5] also combined microwave irradiation with the effect of diphenyl sulfide (DPDS), chemical agent supposed to improve devulcanization process. The authors highlighted the beneficial effects of DPDS on the efficiency of microwave devulcanization process. In another study, Hong et al. [14] also compared devulcanization performance of microwave and ultrasonic procedures and concluded that microwave technique is better than ultrasonic method.

To improve devulcanization efficiency and reduce treatment energy, promising new approach consists of impregnation of the waste rubber with solvent and then heat treating the impregnated material with microwave radiation. In fact, impregnation of the waste rubber could promote the heat rise when exposed to microwave radiation.

Ionic liquids (ILs), considered as an alternative to conventional organic solvents, are of interest in a variety of technological processes. For instance, they were recently used to improve the degree of dispersion of a nanosized $\mathrm{ZnO}$ and Silica in NBR matrix [15]. The desire for green solvents for industrial processes is partially responsible of this keen interest. ILs are (molten) salts composed of large variety of organic cations and organic/inorganic anions [16]. They present several interesting properties: excellent solvent for various organic/inorganic materials, negligible vapor pressure, high thermal and chemical stability, non-flammability, high ionic conductivity, low viscosity and large electrochemical capacitance $[16,17]$. The ionic conduction mechanism is the most important effect in the heating of ILs by microwave; it increases their dielectric loss factor.

In this study, styrene butadiene rubber (SBR) was devulcanized using microwave irradiation and the performance of the devulcanization treatment was analyzed in term of crosslink scission. Effect of $[\mathrm{Pyrr}]\left[\mathrm{HSO}_{4}\right]$, on the devulcanization performance was studied, which represents one of the originalities of this study. 


\section{Experimental}

\subsection{Materials}

The analyzed material in this study was a styrenebutadiene rubber (SBR) obtained by mixing virgin SBR and additive components in an open two roll mill, at room temperature. Parts per hundred rubber [phr] of the whole formulation are reported in Table 1.

The material studied in this work is a mixture of two grades of SBR: $50 \mathrm{phr}$ of SBR1500 with a styrene content of $23.5 \%$ and $50 \mathrm{phr}$ of SBR 1900 with a styrene content of $75 \%$. We deliberately chose such a mixture in order to limit the amount of butadiene, which reduces the number of carbon-carbon double bonds $(\mathrm{C}=\mathrm{C})$ leading to a loosely crosslinked network. Reduction of $\mathrm{C}=\mathrm{C}$ bonds also limits the influence of the external environment (oxygen, ozone ...) on the rubber structure during processing and storage. A loosely cross-linked network facilitates the penetration of solvent through the rubber and therefore improves the sensitivity of methods which allow evaluation of cross-link density and soluble fraction.

Differential scanning calorimetry (DSC) tests were conducted in a nitrogen environment on un-vulcanized and vulcanized SBR, using Netzsch equipment (Netzsch maia 200F, Selb, Germany). Samples weighing about $10 \mathrm{mg}$ were heated from $-100^{\circ} \mathrm{C}$ up to $280^{\circ} \mathrm{C}$ at a rate of $10^{\circ} \mathrm{C} / \mathrm{min}$.

After mixing operation, the rubber was vulcanized at $170^{\circ} \mathrm{C}$ for $7 \mathrm{~min}$, under compression in a hydraulic molding press (Agila, Kortrijk-Bissegem, Bel-

Table 1. Components used for the rubber formulation

\begin{tabular}{|l|c|}
\hline \multicolumn{1}{|c|}{ Components } & $\begin{array}{c}\text { Quantity } \\
\text { [phr] }\end{array}$ \\
\hline Styrene butadiene rubber (SBR 1502) & 50 \\
\hline Styrene butadiene rubber (SS260) & 50 \\
\hline Carbon black (HAF N330) & 60 \\
\hline Naphtenic oil & 25 \\
\hline Paraffinic oil & 1.2 \\
\hline $\begin{array}{l}\text { Rubber antioxidant: N-Isopropyl-N'-phenyl-p- } \\
\text { phenylene-diamine (IPPD) }\end{array}$ & 1.25 \\
\hline $\begin{array}{l}\text { Rubber antioxidant: N-(1,3-Dimethylbutyl)-N'- } \\
\text { phenyl-p-phenylenediamine (6PPD) }\end{array}$ & 1.25 \\
\hline $\begin{array}{l}\text { Rubber antioxidant: 2,2,4-trimethyl-1,2-dihydro- } \\
\text { quinoline (TMQ) }\end{array}$ & 1 \\
\hline Antiozonant agents (Paraffinic wax) & 2 \\
\hline Activator: zinc oxide (ZnO) & 5 \\
\hline $\begin{array}{l}\text { Dispersing agent and accelerator activator: Stearic } \\
\text { acid }\end{array}$ & 2 \\
\hline Vulcanization agent: sulfur (S) & 1.5 \\
\hline $\begin{array}{l}\text { Accelerator: N-cyclohexyl-2-benzothiazole } \\
\text { sulphenamide (CBS) }\end{array}$ & 1.5 \\
\hline
\end{tabular}

gium). Before the devulcanization treatment, the rubber was grounded, at ambient temperature, with a disc mill PQ500 (Phenix Machinery, Sancheville 28800 , France) containing a $4 \mathrm{~mm}$ screen. The obtained ground SBR was passed in a succession of different mesh sieve, which allowed evaluating the particle size distribution of the ground rubber. It appeared that $\sim 96 \%$ of the SBR powder has a size ranging from 1 to $4 \mathrm{~mm}$ and $\sim 4 \%$ has a size lower than $1 \mathrm{~mm}$. The SBR powder of size ranging from 1 to $4 \mathrm{~mm}$ has used in this study.

\subsection{Microwave devulcanization procedure}

A commercial microwave oven (Galanz, Foshan, China) was adapted to be used for devulcanization treatment of SBR ground rubber in laboratory. Stirring system and infrared temperature sensor (SparkFun IR Thermometer Evaluation Board-MLX90614, Lextronic, France) were additionally installed in the oven and the process was controlled by a computer. A mass $M$ of $\sim 50 \mathrm{~g}$ of ground SBR placed in a $250 \mathrm{~mL}$ beaker was exposed to microwave irradiations under magnetron power $P$ of 656 watts, at different time values $t$. The stirring speed was $40 \mathrm{rpm}$. The corresponding microwave specific energy $E$ was evaluated from Equation (1):

$E=\frac{P \cdot t}{M}$

In order to improve the efficiency of the rubber microwave processing, the same procedure was repeated, but by mixing priory the SBR powder in $10 \mathrm{wt} \%$ of ionic liquid pyrrolidinium hydrogen sulfate $[\mathrm{Pyrr}]\left[\mathrm{HSO}_{4}\right]$, in a crystallizer during $10 \mathrm{~min}$ utes. The mixture was left to stand for 1 hour at least, prior to microwave treatment. No specific other treatment was applied for IL and SBR powder before microwave treatment. Properties of this IL are summarized in [18]. It was used because it is a good heat vector and it readily absorbs microwave energy, which could quickly increase the temperature of the mixture during the devulcanization treatment. Moreover, it does not evaporate and does not degrade below $200^{\circ} \mathrm{C}$. Furthermore, this IL is a green solvent with low vapor pressure, high chemical and thermal stability and it is a non-flammable liquid. Thus, the goal of using such IL was to favor the transfer of the microwave energy until the core of the material granulates and to obtain uniform distribution of the temperature through the volume of these particles. 
Table 2. Coding and devulcanization conditions

\begin{tabular}{|l|l|c|c|c|}
\hline $\begin{array}{l}\text { SBR without } \\
{[\text { Pyrr] [HSO }]}\end{array}$ & $\begin{array}{c}\text { SBR with } \\
\mathbf{1 0} \text { wt\% } \\
\text { [Pyrr][HSO }\end{array}$ ] $]$ & $\begin{array}{c}\text { Magnetron } \\
\text { power } \\
{[\mathbf{W}]}\end{array}$ & $\begin{array}{c}\text { Treatment } \\
\text { time } \\
{[\mathbf{s}]}\end{array}$ & $\begin{array}{c}\text { Energy } \\
{[\mathbf{W h} / \mathbf{k g}]}\end{array}$ \\
\hline SBR & SBR-P & 0 & 0 & 0 \\
\hline SBR-110 & SBR-P-110 & 656 & 30 & 110 \\
\hline SBR-220 & SBR-P-220 & 656 & 60 & 220 \\
\hline SBR-330 & SBR-P-330 & 656 & 90 & 330 \\
\hline SBR-440 & SBR-P-440 & 656 & 120 & 440 \\
\hline
\end{tabular}

It was observed that the ground rubber ignited when it was exposed for microwave energy inducing temperature higher than $210^{\circ} \mathrm{C}$. This is probably due to the inflammation of the naphtenic oil (CIRCOSOL 4240) and paraffinic process oil (FLEXON 876) used as plasticizers in the rubber mixture. In fact, the flash points of these oils are 221 and $246^{\circ} \mathrm{C}$, respectively. Thus, the magnetron power value of 656 watts and the maximal time of treatment of $120 \mathrm{~s}$ were selected such as the induced maximal temperature was lower than $\sim 210^{\circ} \mathrm{C}$. The treatment conditions are summarized in Table 2. The SBR soaked in $[\mathrm{Pyrr}]\left[\mathrm{HSO}_{4}\right]$ is denoted SBR-P in this paper.

The heat capacity $C_{\mathrm{p}}$ of the $[\mathrm{Pyrr}]\left[\mathrm{HSO}_{4}\right]$ was determined by the DSC approach. It was achieved by heating a small quantity of IL from ambient temperature to $200^{\circ} \mathrm{C}$ and the heat flow was recorded during the test. Thermo-gravimetric analyses (TGA) were also performed on $[\mathrm{Pyrr}]\left[\mathrm{HSO}_{4}\right]$ using a PerkinElmer Diamond TG/DTA (Waltham, Massachusetts, U.S.A). A small quantity of the liquid was heated from room temperature up to $550^{\circ} \mathrm{C}$, at a rate of $10^{\circ} \mathrm{C} / \mathrm{min}$ and under a nitrogen atmosphere. The mass variation of the liquid was measured as a function of temperature.

\subsection{Evaluation of the devulcanization}

Right after devulcanization treatment, the SBR was washed with distilled water then dried in a vacuum oven (Heraeus Vacuum oven VT6025, Hanau, Germany) at $30^{\circ} \mathrm{C}$ for $24 \mathrm{~h}$. Soluble fraction of ground SBR and devulcanized ground SBR were then determined by extraction in a Soxhlet extractor for $24 \mathrm{~h}$ in acetone (Sigma-Aldrich, Steinheim, Germany) to remove low-molecular weight polar substances like remains of accelerators and curatives. This operation was immediately followed by an extraction for $72 \mathrm{~h}$ in toluene (Sigma-Aldrich, Steinheim, Germany) to remove the non-polar components such as oil and non-crosslinked polymer residues or soluble polymer chains released from the network by the devulcanization process. The soluble fraction $S$ of the material was evaluated using Equation (2):

$S[\%]=\frac{M_{\mathrm{i}}-M_{\mathrm{f}}}{M_{\mathrm{i}}} \cdot 100$

where $M_{\mathrm{i}}$ and $M_{\mathrm{f}}$ are the SBR rubber weight before and after extraction operation respectively.

After swelling in cyclohexane (Sigma-Aldrich, Steinheim, Germany) at room temperature for $72 \mathrm{~h}$, the soluble fraction was weighed and then dried in a vacuum oven at $50^{\circ} \mathrm{C}$ for $24 \mathrm{~h}$. The apparent crosslink density $v_{\mathrm{c}}$ was determined according to Equation (3) [19]:

$\nu_{\mathrm{c}}=\frac{\nu_{\mathrm{r}}+\chi \nu_{\mathrm{r}}^{2}+\ln \left(1-\nu_{\mathrm{r}}\right)}{\nu_{\mathrm{s}}\left(0.5 \nu_{\mathrm{r}}-\nu_{\mathrm{r}}^{1 / 3}\right)}$

with:

$$
\nu_{\mathrm{r}}=\frac{m_{\mathrm{r}}}{m_{\mathrm{r}}+m_{\mathrm{s}}\left(\frac{\rho_{\mathrm{r}}}{\rho_{\mathrm{s}}}\right)}
$$

In Equations (3) and (4), $v_{\mathrm{r}}$ is the volume fraction of the polymer in the swollen specimen, $v_{\mathrm{s}}$ is the molar volume of the solvent, $\chi$ is the Flory-Huggins polymer solvent interaction parameter (equal to 0.489 for SBR-Cyclohexane (ASTM-D6814-02)), $m_{\mathrm{r}}$ is the mass of the dry rubber, $m_{\mathrm{S}}$ is the mass of the solvent absorbed by the rubber, $\rho_{\mathrm{r}}$ and $\rho_{\mathrm{s}}$ are the densities of the dry rubber and solvent respectively.

The percent devulcanization was calculated according to the standard ASTM test method D6814-02, using Equation (5):

Devulcanization $[\%]=\left(1-\frac{\nu_{\mathrm{f}}}{\nu_{\mathrm{i}}}\right) \cdot 100$

where $v_{\mathrm{i}}$ and $v_{\mathrm{f}}$ are the crosslink densities of the samples before and after devulcanization, respectively.

The devulcanization process theoretically results in crosslink scission. However, actual route also causes breaking of principal polymer chains. When this phenomenon is considerable, it induces the loss of the recyclate properties. To further understand the devulcanization mechanism, theoretical relationship developed by Horikx [20] was used, which correlates the rubber soluble fraction of the devulcanizates and the relative decrease in crosslink density. When only main-chain scission takes place, the relative decrease in the apparent crosslink density is given by Equation (6): 
$1-\frac{\nu_{\mathrm{f}}}{\nu_{\mathrm{i}}}=1-\left[\frac{\left(1-\sqrt{S_{\mathrm{f}}}\right)^{2}}{\left(1-\sqrt{S_{\mathrm{i}}}\right)^{2}}\right]$

where $S_{\mathrm{i}}$ and $S_{\mathrm{f}}$ are the soluble fraction of the untreated vulcanizate and reclaimed vulcanizate respectively, $v_{\mathrm{i}}$ is the crosslink density of the untreated vulcanizate and $v_{\mathrm{f}}$ is the crosslink density of the reclaimed vulcanizate.

For pure crosslink scission, the soluble fraction is related to the relative decrease in crosslink density by Equation (7):

$1-\frac{\nu_{\mathrm{f}}}{\nu_{\mathrm{i}}}=1-\left[\frac{\gamma_{\mathrm{f}}\left(1-\sqrt{S_{\mathrm{f}}}\right)^{2}}{\gamma_{\mathrm{i}}\left(1-\sqrt{S_{\mathrm{i}}}\right)^{2}}\right]$

where $\gamma_{\mathrm{i}}$ and $\gamma_{\mathrm{f}}$ are the average numbers of crosslinked units per chain before and after treatment respectively. These parameters were determined from the relation between soluble fraction $S$ and the number of crosslinked units per chain $\gamma$, given by Charlesby [21, 22] under the following form Equation (8):

$S=\frac{1}{(1+\gamma-\gamma s)}$

After applying microwave devulcanization process on SBR soaked in $[\mathrm{Pyrr}]\left[\mathrm{HSO}_{4}\right]$ and no-soaked SBR, glass transition temperature $T_{\mathrm{g}}$ of these materials was determined following the same experimental protocol described above. $T_{\mathrm{g}}$ could be a pertinent indicator of microstructure change which may be caused by, among others, the vulcanization or devulcanization process.

Finally, the Mooney viscosity $\mathrm{ML}(1+4) \cdot 100^{\circ} \mathrm{C}$ was measured using a Mooney viscometer from Gibitre Instruments (Bergamo, Italy).

All specimens were continuously stored under controlled temperature of $4^{\circ} \mathrm{C}$.

\section{Results and discussion}

Figure 1 shows the heat flow recorded during heating, as a function of temperature, for un-vulcanized and vulcanized SBR. It exhibited an endothermic transition characteristic of glass transition temperature, $T_{\mathrm{g}}$, estimated at $-48^{\circ} \mathrm{C}$ for the raw SBR and at $-43^{\circ} \mathrm{C}$ for the vulcanized SBR. The increase of $T_{\mathrm{g}}$ from -48 to $-43^{\circ} \mathrm{C}$ confirmed the successful vulcanization of the studied material. In fact, the vulcanization treatment led to the formation of 3D network, thereby reducing macromolecular mobility resulting in augmentation of $T_{\mathrm{g}}$. Moreover, an exo-

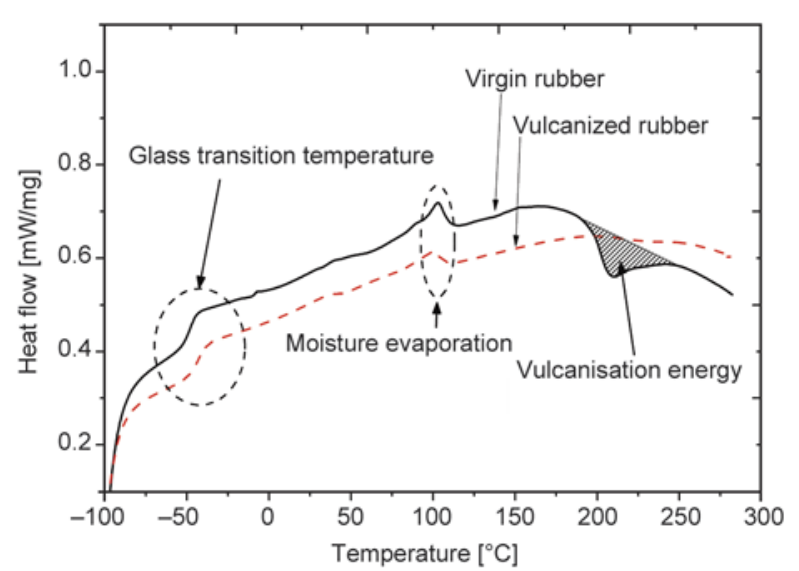

Figure 1. DSC curves of the SBR before and after vulcanization

thermic peak beginning at $\sim 160^{\circ} \mathrm{C}$ and reaching its maximum at $\sim 210^{\circ} \mathrm{C}$, was observed. This peak corresponds to the temperature of the SBR vulcanization reaction. Such a transition was not present in the vulcanized rubber thermogram, thus, further proving that the vulcanization of the material was entirely accomplished.

The heat capacity $C_{\mathrm{pe}}$ of $[\mathrm{Pyrr}]\left[\mathrm{HSO}_{4}\right]$ is reported in Figure 2 as a function of temperature. This heat capacity is evaluated from Equation (9):

$C_{\mathrm{pe}}=\frac{m_{\mathrm{r}}}{m_{\mathrm{e}}} \cdot \frac{\varphi_{\mathrm{e}}-\varphi_{0}}{\varphi_{\mathrm{r}}-\varphi_{0}} \cdot C_{\mathrm{pr}}$

where $m_{\mathrm{r}}$ and $m_{\mathrm{e}}$ are masses of aluminum oxide and ionic liquid $[\mathrm{Pyrr}]\left[\mathrm{HSO}_{4}\right]$ respectively $; \varphi_{0}, \varphi_{\mathrm{r}}$ and $\varphi_{\mathrm{e}}$ are heat flows measured for empty crucible, aluminum oxide and IL respectively; $C_{\mathrm{pr}}$ represent mass calorific capacities of aluminum oxide. The heat capacity of distilled water was also measured and showed in Figure 2. It can be seen that $C_{\mathrm{p}}$ of [Pyrr] $\left[\mathrm{HSO}_{4}\right]$ was constant up to $120^{\circ} \mathrm{C}$ and was

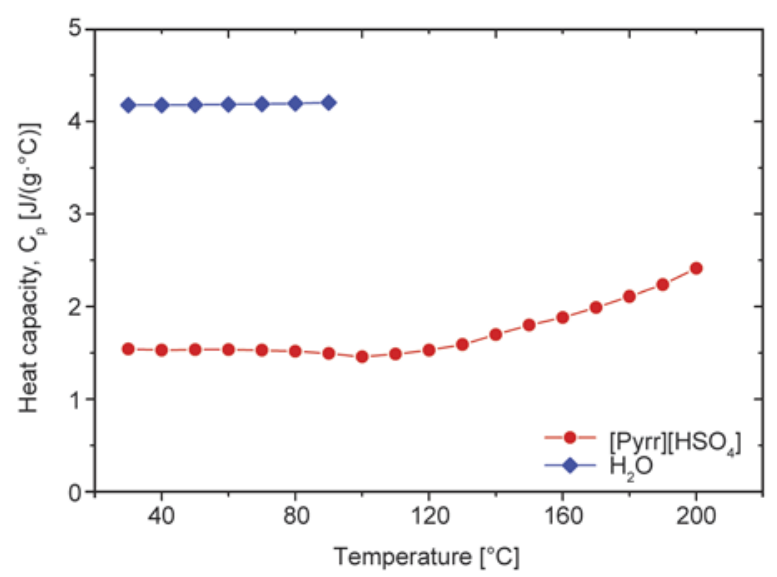

Figure 2. Heat capacity of $[\mathrm{Pyrr}]\left[\mathrm{HSO}_{4}\right]$ and water as a function of temperature 


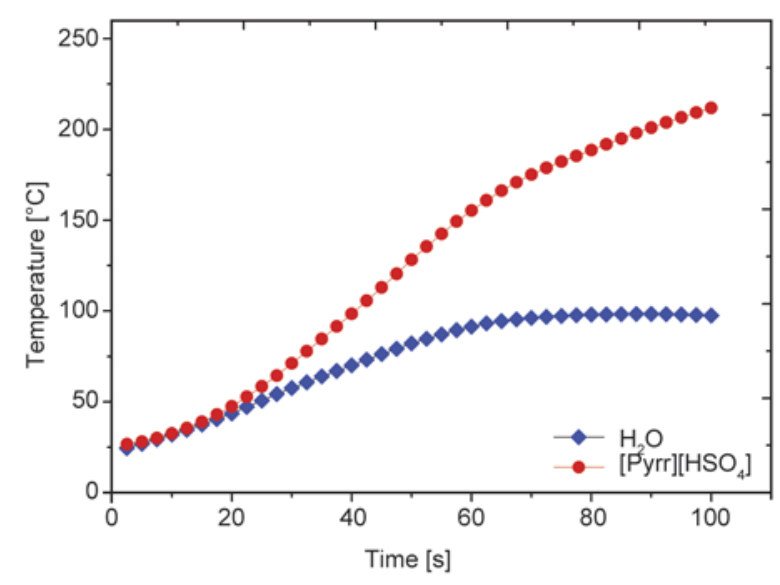

Figure 3. Temperatures of water and $[\mathrm{Pyrr}]\left[\mathrm{HSO}_{4}\right]$ as a function of microwave irradiation time

three times smaller than that of distilled water, thus, proving a good heat transfer capacity of this IL.

Figure 3 presents temperature evolutions of $[\mathrm{Pyrr}]\left[\mathrm{HSO}_{4}\right]$ and distilled water as a function of the time, recorded under microwave irradiation at magnetron power of $656 \mathrm{~W}$. It was evident that the temperature of the IL increased more quickly than water to become two times greater after $100 \mathrm{~s}$ of exposition. Moreover, contrary to the temperature of the water that stabilized at $100^{\circ} \mathrm{C}$ beyond $80 \mathrm{~s}$, the temperature of the IL increased continuously over the range of analysis time. These results further confirmed the relative high ability of [Pyrr] $\left[\mathrm{HSO}_{4}\right]$ to convert microwave energy in heat, compared to water.

Figure 4 shows the weight loss of $[\mathrm{Pyrr}]\left[\mathrm{HSO}_{4}\right]$ as a function of temperature, obtained by TGA measurements. The curve exhibited a first plateau followed by a sudden decrease corresponding to its thermal degradation. The onset degradation temperature, $T_{\text {onset, }}$ was evaluated by the method of tangent and

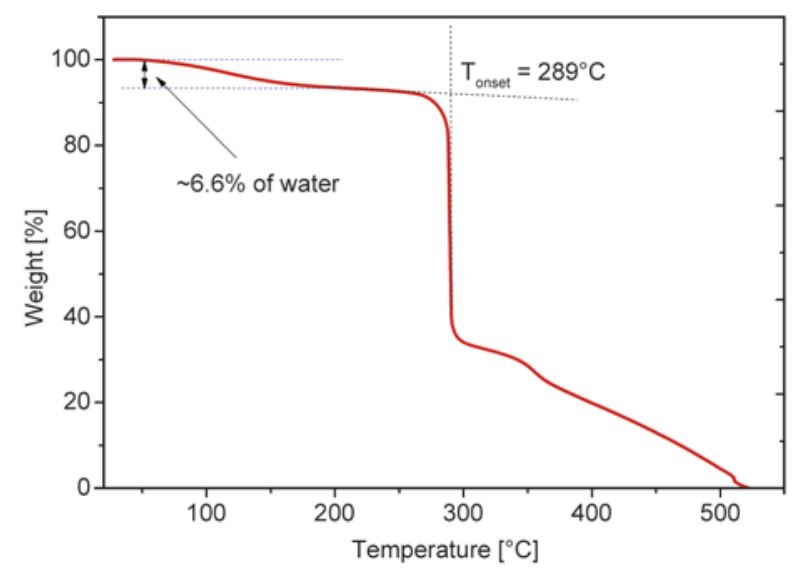

Figure 4. TGA curve of $[\mathrm{Pyrr}]\left[\mathrm{HSO}_{4}\right]$

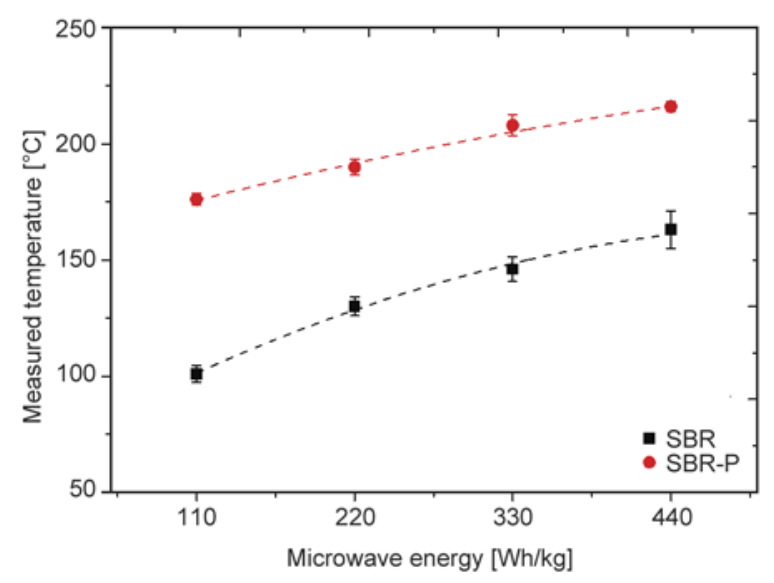

Figure 5. Temperature of ground rubber with and without ionic liquid as a function of microwave energy

was found to be $\sim 289^{\circ} \mathrm{C}$, as shown in Figure 4 . To avoid the degradation of [Pyrr] $\left[\mathrm{HSO}_{4}\right]$ during the devulcanization treatment, this temperature value should not be exceeded when the SBR rubber combined with $[\mathrm{Pyrr}]\left[\mathrm{HSO}_{4}\right]$ is treated by microwave irradiation as described above.

Figure 5 shows the evolution of the temperature reached by rubber powder exposed to microwave irradiation, for different energy values. The presence of ionic liquid [Pyrr] $\left[\mathrm{HSO}_{4}\right]$ significantly favored the material heating over the whole range of the considered microwave energy values. In fact, the temperature reached by the SBR soaked in $[\mathrm{Pyrr}]\left[\mathrm{HSO}_{4}\right]$ was 1.70 times greater than the dry SBR. This result suggested that $[\mathrm{Pyrr}]\left[\mathrm{HSO}_{4}\right]$ could play energy vector role during the microwave devulcanization process.

Figure 6 shows evolutions of the soluble fractions (Equation (2)) of treated rubber as a function of microwave energy. The soluble fraction indicates the extent to which the rubber network is broken. From the Figure 6, it was evident that microwave treatment of sulfur-cured SBR exhibited a soluble fraction similar up to $220 \mathrm{Wh} / \mathrm{kg}$, irrespective of IL soaking. This indicated that up to this treatment energy value, rubber network was still intact. Beyond this threshold point, the soluble fraction sharply increased with increasing devulcanization microwave energy i.e. by increasing the treatment temperature. The increase was more significant for the powder mixed with [Pyrr] $\left[\mathrm{HSO}_{4}\right]$, further proving the positive role played by the IL in the microwave devulcanization process of rubbers.

Figure 7 depicts crosslink densities (Equation (3)) of SBR and SBR-P samples, as a function of micro- 


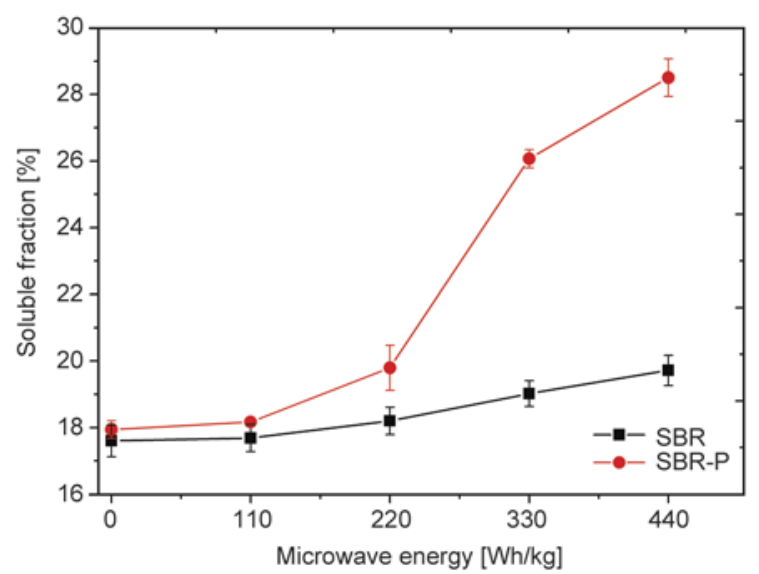

Figure 6. Soluble fraction of ground rubber with and without ionic liquid as a function of microwave energy

wave energy. The crosslink density decreased slightly and slowly with the microwave energy up to $220 \mathrm{Wh} / \mathrm{kg}$, critical value beyond which the crosslink density decreased significantly for the SBR-P rubber. These results agreed well with the findings shown in Figure 6 and confirmed that the liquid ionic $[\mathrm{Pyrr}]\left[\mathrm{HSO}_{4}\right]$ favored the microwave devulcanization process. Figure 8 also shows the devulcanization proportions (Equation (5)) of the SBR and SBR-P, as a function of microwave energy. Curves showed the same trends as soluble fractions (Figure 6), confirming existence of a relationship between the two quantities (Equations (2) and (5)). Devulcanization fractions are also represented in Figure 9 as a function of average temperature generated in rubber powder, measured with infrared sensor during microwave irradiation. It should be noticed that rubber powder was mixed with an agitator during the devulcanization treatment. The experimental data of both SBR and SBR-P were fitted by power laws. It was evident that for a given microwave energy

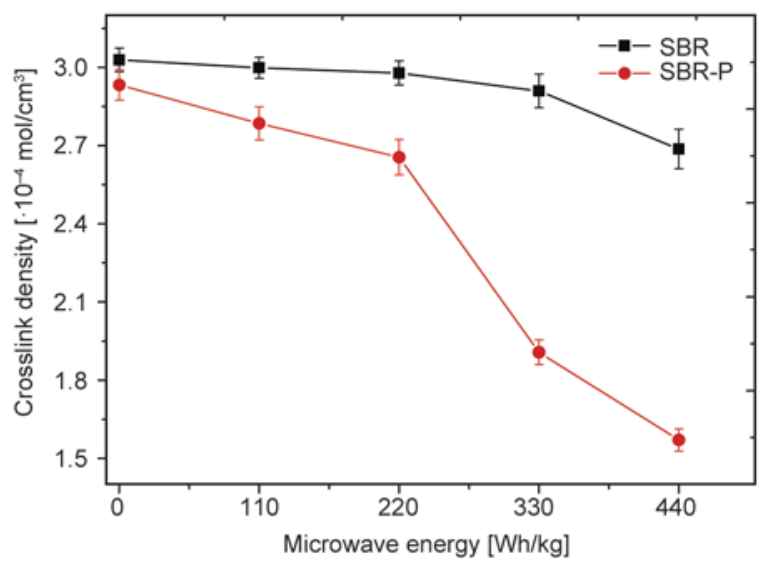

Figure 7. Crosslink density of ground rubber with and without ionic liquid as a function of microwave energy

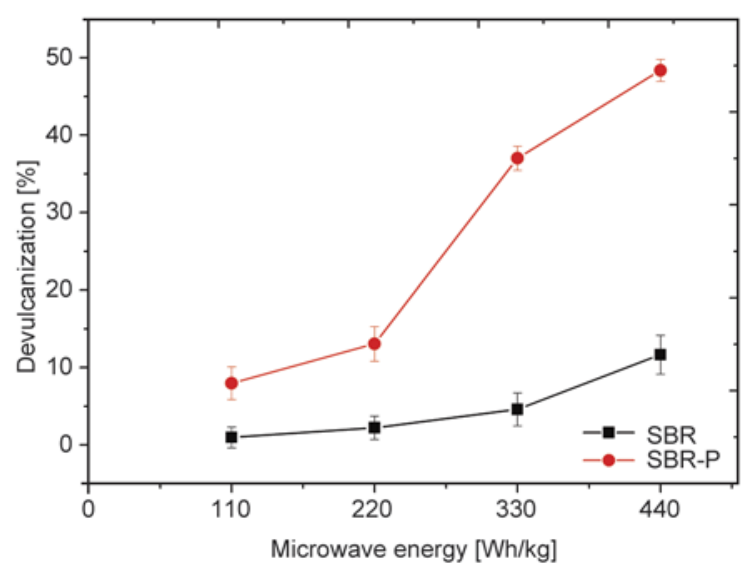

Figure 8. Devulcanization of ground rubber with and without ionic liquid as a function of microwave energy

value, average temperature reached by SBR-P was much higher than the one reached by dry SBR. Also, the devulcanization process seemed to be efficient above a temperature threshold of $\sim 200^{\circ} \mathrm{C}$, in agreement with the results found by Saiwari et al. [23] in the devulcanization of SBR in an internal mixer with addition of treated distillate aromatic extract (TDAE) oil and diphenyldisulfide (DPDS). To further analyze the devulcanization of rubbers by microwave process, the distribution of the treatment temperature was recorded with an infrared camera FLIR JADE-MWIR, in both SBR and SBR-P, after subjecting them to microwave energy. The obtained micrographs are shown in Figure 10, as an example for treatment energy of $40 \mathrm{Wh} / \mathrm{kg}$. The temperature was observed to be more homogeneous and better diffused in the SBR-P powder than in the dry SBR powder. This result proved that microwave irradiation produced efficient internal heating by direct coupling of microwave energy with the IL, resulting in higher average temperature and homo-

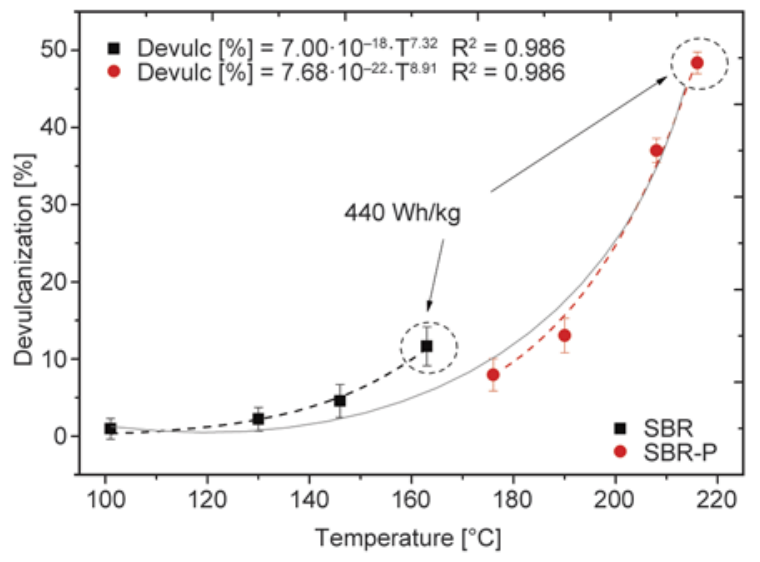

Figure 9. Devulcanization of ground rubber with and without ionic liquid as a function of temperature reached during microwave treatment 


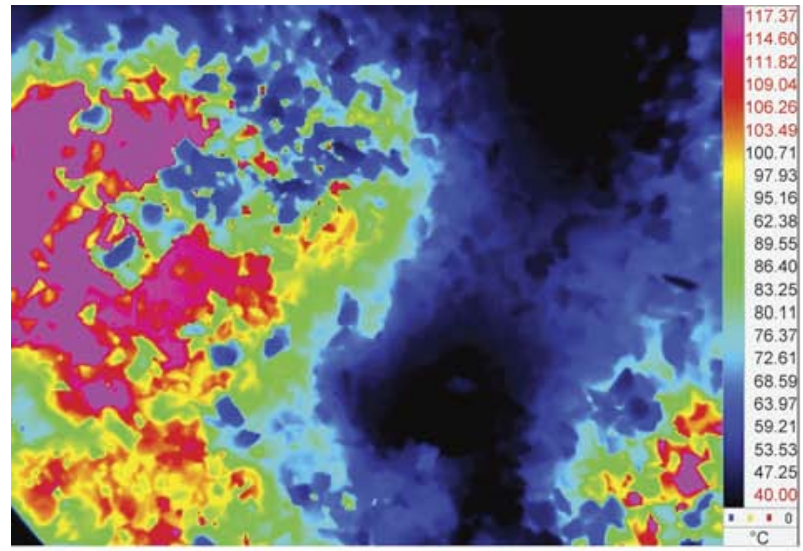

a)

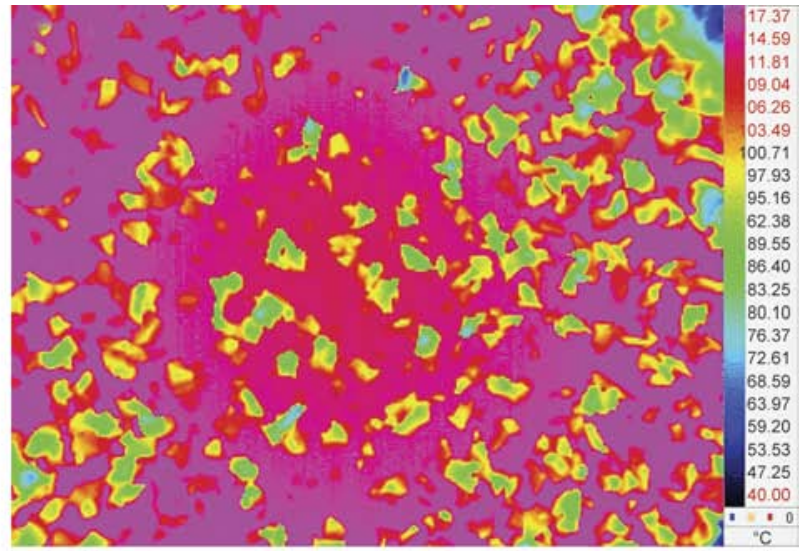

b)

Figure 10. Temperature of the ground rubber (a) without ionic liquid and (b) with ionic liquid, measured with a thermal camera

geneous heating throughout the treated material, which can partially explain the lower crosslinking density of the SBR-P shown in Figure 7.

Figure $11 \mathrm{a}$ and $11 \mathrm{~b}$ show the experimentally determined soluble fractions of SBR and SBR-P respectively, as a function of devulcanization fractions evaluated using Equation (5), for different microwave energy values. Theoretical curves of Horikx given by Equations (6) and (7) are also plotted in Figure 11. The experimental data fitted well with the Horikx's curve corresponding to the crosslink scission (Equation (7)), suggesting that devulcanization process occurred in all microwave treated materials by rather selective breakage of crosslinks.

However, magnitude of this devulcanization was higher as the microwave energy was increased and when the SBR was imbibed in IL. An increase in the microwave energy resulted in a shift of the data point to the right hand region of the graph which indicated decrease of crosslink density. The shift phenomenon was even more pronounced for the ground rubber mixed with IL. For instance, for the same microwave energy of $440 \mathrm{Wh} / \mathrm{kg}$, apparent devulcanization in SBR-P reached $\sim 50 \%$, which was four times greater than dry SBR.

Figure 12 shows the evolution of $T_{\mathrm{g}}$ for the SBR and SBR-P, as a function of microwave energy. The $T_{\mathrm{g}}$ of the SBR remained almost constant in the whole range of the applied microwave energy values, suggesting that there was no significant change in the material microstructure in this range. However, a significant and continuous increase in $T_{\mathrm{g}}$ with microwave energy values was observed for SBR-P, indicating that the structure changed as the microwave energy was enhanced. The increase of the $T_{\mathrm{g}}$ has also been reported by others authors for devulcanization of sulfur vulcanized rubber by ultrasound [24]. This change in $T_{\mathrm{g}}$ was attributed to the formation of cyclic sulfur structures in the polymer chains. Such a structure limits the mobility of the chains, increasing the $T_{\mathrm{g}}$ of the elastomer. For microwave devulcanization of ground tire rubber [25], this dif-

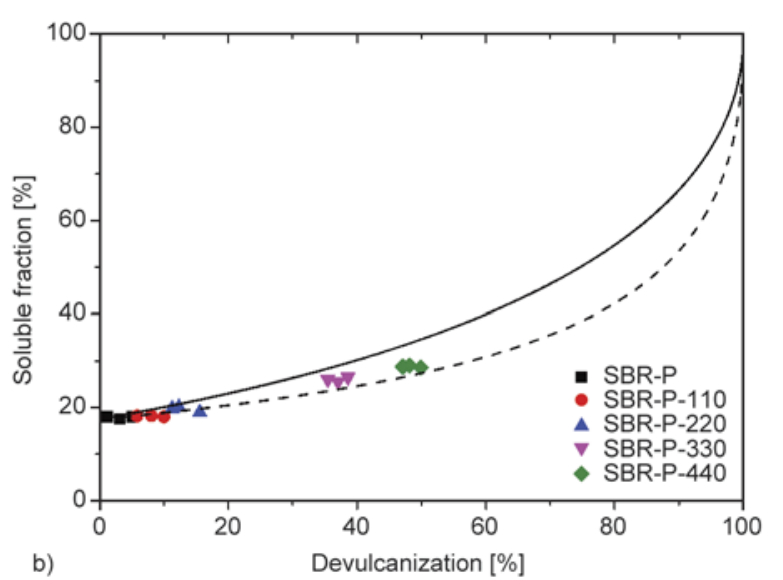

Figure 11. Horikx diagram for ground rubber (a) without ionic liquid and (b) with ionic liquid 


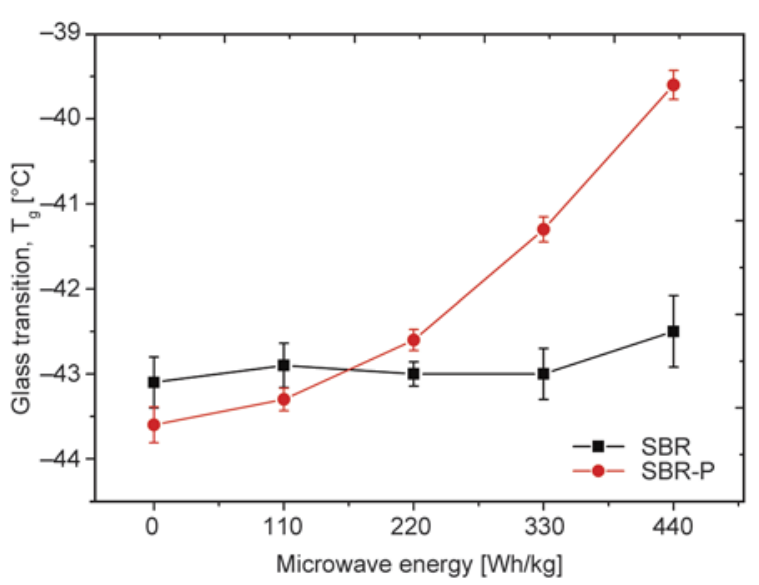

Figure 12. Glass transition temperatures of ground rubber with and without ionic liquid as a function of microwave energy

ference in the $T_{\mathrm{g}}$ values was also attributed to the higher carbon black content of the devulcanized samples. On the other hand, the volatilization at high temperature (above $200^{\circ} \mathrm{C}$ ) of the extender oil which works as a plasticizer for the rubber molecules also tends to diminish the molecular mobility of the rubber, increasing its $T_{\mathrm{g}}$.

Gums were generated from the devulcanized SBR and SBR-P materials, treated with microwave energy of $440 \mathrm{Wh} / \mathrm{kg}$. To do this, a laboratory two-roll mill was used with the friction ratio of 1.3 , at temperature of $50^{\circ} \mathrm{C}$ and mixing time of $10 \mathrm{~min}$ for each blend.

The soluble fractions and the devulcanization proportions of the obtained mixtures are reported in Horikx diagram in Figure 13. Data of microwave treated SBR powder are also plotted in this figure. It can be observed that experimental points of mixtures shifted significantly to the right region of the Figure 13, while remaining well fitted by theoretical curve corre-

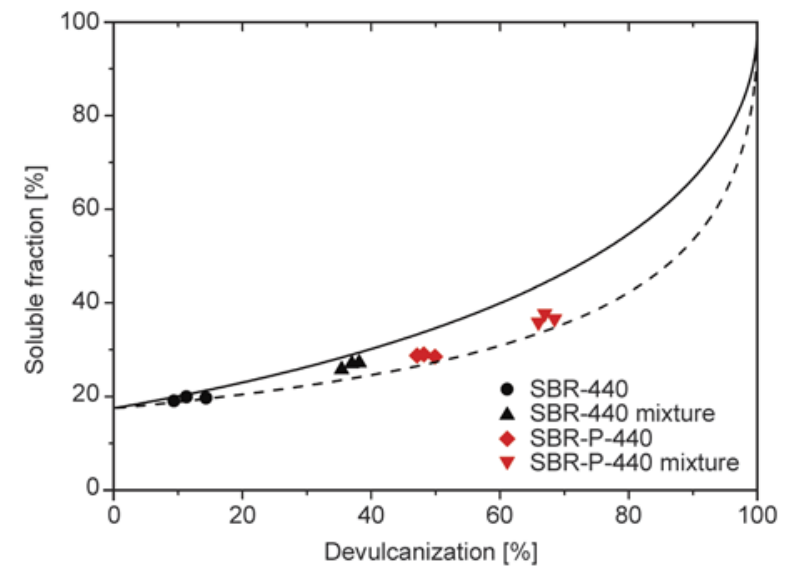

Figure 13. Horikx diagram for SBR powder and SBR mixture

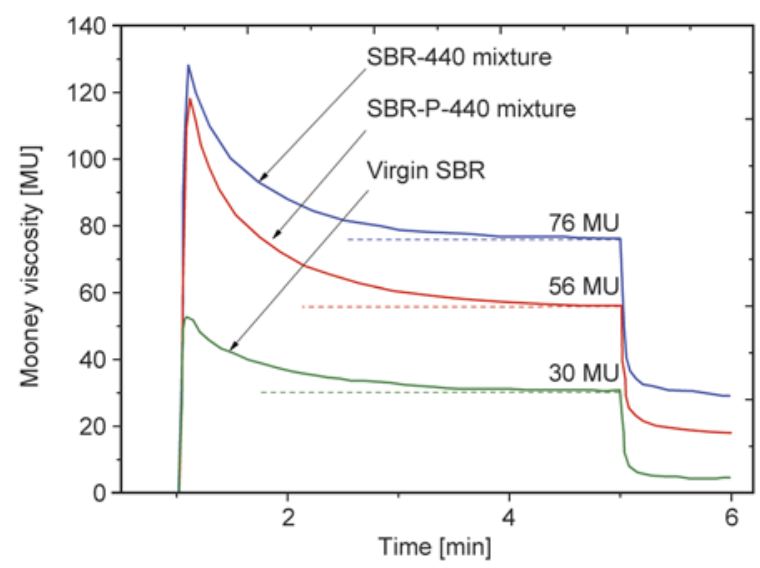

Figure 14. Mooney viscosity $\mathrm{ML}(1+4) 100^{\circ} \mathrm{C}$ for unvulcanized raw rubber and different recycled rubbers (MU is Mooney unit of viscosity: $1 \mathrm{MU}=$ $0.083 \mathrm{~N} \cdot \mathrm{m})$

sponding to the main-chain scission (Equation (7)). These points moved from 13 to $38 \%$ and from 50 to $65 \%$ for SBR and SBR-P respectively. This result indicated that the mechanical shearing of rubber, involved herein into the two-roll mill, favored the devulcanization process, suggesting that combining mechanical loading with microwave energy and IL could be a most profitable procedure allowing an optimal devulcanization of rubbers.

Figure 14 shows the Mooney viscosity and Mooney relaxation of virgin unvulcanized rubber and reclaimed rubber obtained after ground rubber mixing. The Mooney viscosity of the rubber gives an indication of the relative devulcanization degree. Indeed, more is the degree of devulcanization, lower is the Mooney viscosity [26]. Thus, curves in Figure 14 confirmed the earlier results. The specimen generated from the ground rubber previously treated by microwave in the presence of IL exhibited Mooney viscosity close to that of the virgin rubber, proving high devulcanization of this specimen.

\section{Conclusions}

Devulcanization styrene butadiene rubber (SBR) by microwave irradiations has been studied. The effect of IL (pyrrolidinium hydrogen sulfate [Pyrr] $\left[\mathrm{HSO}_{4}\right]$ ) on this devulcanization was particularly analyzed. High ability of this IL to convert microwave energy in heat has been proved. The increase in the soluble fraction with increasing microwave energy was also more significant for the powder mixed with $[$ Pyrr $]\left[\mathrm{HSO}_{4}\right]$, further confirming the positive role of the IL in the microwave devulcanization process of rubbers. The measured crosslink density decreased 
with the microwave energy, more significantly for the SBR-P, testifying that the IL favored the microwave devulcanization mechanism. Results highlighted a relationship between the soluble fraction and the devulcanization quantities. In fact, the devulcanization evaluated from Equation (5) was also higher as the microwave energy was increased and when the SBR was impregnated in IL, confirming again the positive role of this IL in the devulcanization treatment. Analysis of the temperature distribution through the ground rubber proved that microwave irradiation produced efficient heating when microwave energy was coupled with the IL. This could explain the better devulcanization of the rubber in presence of the IL. Finally, the experimental data fitted well with the Horikx's curve corresponding to the crosslink scission, suggesting that devulcanization process occurred in all microwave treated materials by rather selective breakage of crosslinks. The magnitude of this devulcanization was higher as the microwave energy was increased and when the rubber was imbibed in IL.

As a main conclusion, the use of IL exhibits strong potential towards successful and efficient devulcanization of rubbers and reduce the energy required for devulcanization treatment.

\section{Acknowledgements}

The authors thank the society Phenix Industries from Sancheville (28800), France, for its technical help.

\section{References}

[1] Fukumori K., Matsushita M., Mouri M., Okamoto H., Sato N., Takeuchi K., Suzuki Y.: Dynamic devulcanization and dynamic vulcanization for recycling of crosslinked rubber. Kautschuk Gummi Kunststoffe, 59, 405-411 (2006).

[2] Sun X., Isayev A. I.: Ultrasound devulcanization: Comparison of synthetic isoprene and natural rubbers. Journal of Materials Science, 42, 7520-7529 (2007).

DOI: $10.1007 / \mathrm{s} 10853-007-1623-9$

[3] Sun X., Isayev A. I.: Continuous ultrasonic devulcanization: Comparison of carbon black filled synthetic isoprene and natural rubbers. Rubber Chemistry and Technology, 81, 19-46 (2008).

DOI: $10.5254 / 1.3548195$

[4] Novotny D. S., Marsh R. L., Masters F. C., Tally D. N.: Microwave devulcanization of rubber. U.S. Patent 4104205 A, USA (1978).
[5] Vega B., Montero L., Lincoln S., Agulló N., Borrós S.: Control of vulcanizing/devulcanizing behavior of diphenyl disulfide with microwaves as the heating source. Journal of Applied Polymer Science, 108, 19691975 (2008).

DOI: $10.1002 /$ app. 27578

[6] De D., De D., Singharoy G. M.: Reclaiming of ground rubber tire by a novel reclaiming agent. I. Virgin natural rubber/reclaimed GRT vulcanizates. Polymer Engineering and Science, 47, 1091-1100 (2007). DOI: 10.1002/pen.20790

[7] Rajan V. V., Dierkes W. K., Joseph R., Noordermeer J. W. M.: Recycling of NR based cured latex material reclaimed with 2,2'-dibenzamidodiphenyldisulphide in a truck tire tread compound. Journal of Applied Polymer Science, 102, 4194-4206 (2006).

DOI: $10.1002 /$ app. 24563

[8] Tsuchii A., Takeda K.: Rubber-degrading enzyme from a bacterial culture. Applied and Environmental Microbiology, 56, 269-274 (1990)

[9] Karger-Kocsis J., Mészáros L., Bárány T.: Ground tyre rubber (GTR) in thermoplastics, thermosets, and rubbers. Journal of Materials Science, 48, 1-38 (2013). DOI: $10.1007 / \mathrm{s} 10853-012-6564-2$

[10] Fix S. R.: Microwave devulcanization of rubber. Elastomerics, 112, 38-40 (1980).

[11] Bani A., Polacco G., Gallone G.: Microwave-induced devulcanization for poly(ethylene-propylene-diene) recycling. Journal of Applied Polymer Science, 120, 2904-2911 (2011).

DOI: 10.1002/app.33359

[12] Landini L., de Araújo S. G., Lugão A. B., Wiebeck H.: Preliminary analysis to BIIR recovery using the microwave process. European Polymer Journal, 43, 27252731 (2007).

DOI: $10.1016 /$ j.eurpolymj.2007.03.017

[13] Scagliusi S. R., Araújo S. G., Landini L., Lugão A. B.: Study of properties of chloroprene rubber devulcanizate by radiation in microwave. in 'International Nuclear Atlantic Conference 2009. Rio de Janeiro, Brazil - INAC' p.8 (2009).

[14] Hong Y. J., Jeong K. M., Saha P., Suh J., Kim J. K.: Processing and characterization of microwave and ultrasonically treated waste-EPDM/LDPE polymer composites. Polymer Engineering and Science, 55, 533-540 (2014).

DOI: $10.1002 /$ pen.23916

[15] Maciejewska M., Zaborski M.: Effect of ionic liquids on the dispersion of zinc oxide and silica nanoparticles, vulcanisation behaviour and properties of NBR composites. Express Polymer Letters, 8, 932-940 (2014). DOI: $10.3144 /$ expresspolymlett.2014.94

[16] Armand M., Endres F., MacFarlane D. R., Ohno H., Scrosati B.: Ionic-liquid materials for the electrochemical challenges of the future. Nature Materials, 8, 621629 (2009).

DOI: $10.1038 /$ nmat2448 
[17] Silvester D. S., Rogers E. L., Compton R. C., Mckenzie K. J., Ryder K. S., Endres F., Macfarlane D., Abbott A. P.: Technical aspects. in 'Electrodeposition from ionic liquids' (eds.: Enders F., MacFarlane D. R., Abbot A. P.) Wiley-VCH, Weinheim, 287-351 (2008).

[18] Anouti M., Jacquemin J., Porion P.: Transport properties investigation of aqueous protic ionic liquid solutions through conductivity, viscosity, and NMR selfdiffusion measurements. Journal of Physical Chemistry B, 116, 4228-4238 (2012).

DOI: $10.1021 /$ jp3010844

[19] Flory P. J.: Molecular size distribution in three dimensional polymers. III. Tetrafunctional branching units. Journal of the American Chemical Society, 63, 30963100 (1941).

DOI: $10.1021 / \mathrm{ja} 01856 \mathrm{a} 063$

[20] Horikx M. M.: Chain scissions in a polymer network. Journal of Polymer Science, 19, 445-454 (1956). DOI: $10.1002 /$ pol.1956.120199305

[21] Charlesby A.: Solubility and molecular size distribution of crosslinked polystyrene. Journal of Polymer Science, 11, 513-520 (1953). DOI: $10.1002 /$ pol.1953.120110601
[22] Charlesby A.: Gel formation and molecular weight distribution in long-chain polymers. Proceedings of the Royal Society of London A, 222, 542-557 (1954). DOI: $10.1098 /$ rspa.1954.0094

[23] Saiwari S., Dierkes W. K., Noordermeer J. W. M.: Comparative investigation of the devulcanization parameters of tire rubbers. Rubber Chemistry and Technology, 87, 31-42 (2013).

DOI: $10.5254 /$ rct.13.87933

[24] Levin V. Y., Kim S. H., Isayev A. I.: Effect of crosslink type on the ultrasound devulcanization of SBR vulcanizates. Rubber Chemistry and Technology, 70, 641-649 (1997).

DOI: $10.5254 / 1.3538450$

[25] Scuracchio C. H., Waki D. A., da Silva M. L. C. P.: Thermal analysis of ground tire rubber devulcanized by microwaves. Journal of Thermal Analysis and Calorimetry, 87, 893-897 (2007).

DOI: $10.1007 / \mathrm{s} 10973-005-7419-8$

[26] Sutanto P.: Development of a continuous process for EPDM devulcanization in an extruder. PhD Thesis, University Library Groningen, Netherlands (2006). 